\title{
A Systematic Evaluation OF MANET ROUTING PROTOCOLS OVER UDP AND TCP IN MULTI-HOP WIRELESS NETWORK
}

\author{
Adebayo Seyi ${ }^{1}$ and Ogunseyi Taiwo ${ }^{2}$ \\ ${ }^{1}$ Department of Information and Communication Engineering, China University \\ of Mining and Technology, Xuzhou, China \\ ${ }^{2}$ Department of Information Security, Communication University of China, \\ Beijing, China
}

\begin{abstract}
There are genuine concerns for the right transport connection to be deployed on a particular routing protocol in order to have a reliable, fast and robust communication in spite of the size and the dynamics of the mobile ad-hoc network (MANET) topology. This paper comparatively studies the individual implementation of reactive and proactive protocols on both UDP and TCP transport connection using packet delivery ratio (PDR), throughput, end to end delay and delay variation (jitter) as quality of service $(Q o S)$ metrics.

We studied the combination of both the transport connection and routing protocol that will deliver the best QoS in simple and complex network scenarios with source and destination nodes fixed and the intermediate nodes randomly moving throughout the simulation time. More so, the intrinsic characteristics of the routing protocols regarding the QoS metrics and transport connection are studied. Forty simulations were run for simple and complex multi-hop network models and the results were analyzed and presented.
\end{abstract}

\section{KEYWORDS}

MANET, Proactive, Reactive, QoS, UDP, TCP

\section{INTRODUCTION}

Mobile ad-hoc networks (MANETs) are wireless ad-hoc nodes that form a communication network without cellular base stations, access points or any centralized infrastructure [1]. The participating nodes carry out the tasks of network management, security and resource allocation.

Extensive researches have been carried out in MANETs and challenges like security, collision avoidance, redundant links, multi-hop routing and synchronization have not been completely solved. For instance, to solve routing problem in networks that use proactive routing, every node in the network must share its routing table with the neighboring nodes in spite of the state of the network (active or passive). This causes large overhead, excess bandwidth usage and increased power dissipation, thus there is always need for a trade-off.

Furthermore, MANET promises good alternative to several mobile nodes to communicate and exchange data. The infrastructure-less and decentralized nature of MANET makes it easily deployable in military exercises, underground mining operation, disaster relief operations and in any other scenario where traditional cellular network is inaccessible.

Natarajan Meghanathan et al. (Eds) : ACITY, AIAA, DPPR, CNDC, WIMNET, WEST, ICSS - 2019

pp. 171-182, 2019. @ CS \& IT-CSCP 2019

DOI: $10.5121 /$ csit.2019.91717 
However, the choice of transport connection (connection-oriented or connectionless) to use on a routing protocol that will deliver secure, reliable and fast data sometimes gives a challenge, also bandwidth efficiency, congestion control and power consumption of these connections are considered. This has led many researchers to propose other variants of the connection-oriented TCP transport protocols like TCP/Reno, TCP/Tahoe, TCP/Vegas, TCP/Westwood, FACK, TCP/Newreno, SACK - the performance of these variants are studied in different scenarios by [2], [3], [4] and variant of connectionless UDP transport protocol like UDP-Lite has also been proposed.

More so, this can also be said of routing protocols - recently, researchers have proposed several new routing protocols and the existing ones are improved continuously to better meet the challenges in data communication in different network conditions.

\section{RELATED WORKS}

A related research was performed by [5], the authors studied the effects of load and density on DSDV and AODV routing protocols using both UDP/CBR and TCP/FTP traffics. They evaluated the throughput, PDR, and end-to-end delay under different network conditions and concluded that AODV performed better in PDR while end-to-end delay is minimal in DSDV. They both perform poorly with high density and load.

Authors in [6] compared three MANET routing protocols (DSR, DSDV and AODV) based on Random Waypoint Mobility Model, they studied how the protocols compare in some selected QoS metrics like normalized routing load (NRL) amongst others. It was also concluded that DSDV, which is a proactive protocol has best performance in end-to-end delay while the other reactive protocols performed better in other QoS metrics.

In addition, MANET routing protocols' performance for video streaming was studied by authors in [7] and it was concluded that Enhanced Video Streaming in MANET (EVSM) performed better than AODV and AOMDV with the explanation that in EVSM, routing method (i.e. 60:40 Multipath Routing Design) offered a preferable performance over other studied protocols. The performance assessment parameters used in this paper are: PDF/PDR, delay, throughput and routing overhead.

Moreover, authors in [8] studied a comprehensive performance analysis of MANET protocols. The compared protocols are reactive (DSR, AODV) and proactive (DSDV) protocols using CBR and TCP as transport connections. The authors concluded that for CBR traffic, AODV performs better than the other studied routing protocols in packet delivery fraction (PDF) and also its delay is low when compared to DSDV and DSR. In TCP traffic, AODV performs lower than DSDV and its performance is comparable with DSR in packet delivery factor (PDF).

In this research work, Destination Sequenced Distance Vector (DSDV), Dynamic Source Routing (DSR), Ad-hoc on Demand Distance Vector (AODV) and Ad-hoc on Request Multipath Distance Vector (AOMDV) with different node numbers are studied. The protocols' functionalities and quality of service (QoS) delivery are studied individually through simulation on two higher layer transport protocols: user datagram protocol (UDP) and transmission control protocol (TCP).

During simulation, file transfer protocol (FTP) traffic generation module was attached to the TCP agent to generate data while UDP data was generated using constant-bit rate (CBR). The study was in both simple and complex environment with the mobile nodes randomly moving at $15 \mathrm{~m} / \mathrm{s}$ to $70 \mathrm{~m} / \mathrm{s}$. 
Both the source nodes (srcNode) and the destination nodes (dstNode) were immobile and placed in an adjacent position to each other.

\section{Outlines of the Routing Protocols}

\section{A. Ad-Hoc on Demand Distance Vector}

This protocol works base on destination sequence number in DSDV and route discovery in DSR [8]. By means of the destination sequence numbers, the process of AODV is loop-free; it also circumvents Bellman-Ford "counting to infinity" challenge, which thus gives it rapid convergence when ad-hoc network topology changes [9]. Unlike DSR, AODV packet size is even and it maintains a single route for a source-destination pair [10].

In order to establish a connection, the source node $S$ will first browse its routing table for a route to destination (D), if there is no route, the source (S) will broadcast a RREQ message to all active nodes, a node accepts the request and adds its own address to the packet only if it has an updated route to the destination node or it is the destination node [11, 12]. A multiple RREP are sent back to $S$ and it chooses the path with the greatest sequence number and smallest hop sum to the destination (D). Source S starts the route by sending a multicast activation (MACT) to the next hop.

To detect link breakage, nodes periodically exchange HELLO messages among themselves. Continuous nodes' movements and link breakage are sent to the Source node using Route Error (RERR) messages, which also maintains that the destination sequence numbers are up-to-date [13].

\section{B. Ad-hoc on Request Multipath Distance Vector (AOMDV)}

It is an extension of AODV for computing multiple loop-free as well as link disjoint paths. It recovers faster from link failure due to multipath routing capability.

AOMDV routes are established on-demand and it has more message overheads during route discovery because of its multi-route feature. Nonetheless, setting up several paths has assured desirable results in the security of the transmitted packets. Using multiple path protocol enhances the session security by ensuring intermediate nodes have only one path passing through them [14]. Also, due to it multipath nature it can detect any form of impersonation attacks like session hijacking, gray-hole attack, black hole attack and spoofing by authenticating the real identity of the destination node through another path.

\section{Dynamic Source Routing (DSR)}

Like AODV, it forms a route on-demand when an active node requests a connection. It has route detection and route maintenance capabilities that work together to permit the detection and maintenance of source routes. Each mechanism operates entirely on demand. DSR requires no periodic exchange of packets of any kind in the network. [15]. More so, DSR has a benefit of storing multiple routes in it route cache before starting route discovery and if route is found there is no need for route discovery. Route Caching in DSR is also used if there is a link failure. Should there be any failure, other route in the local cache can be used provided that there is route to D, and if otherwise, source $S$ starts new route discovery by sending RRER message thus caching enhances route discovery and easy propagation of RREQ. 


\section{Distance Sequence Distance Vector (DSDV)}

It is a proactive protocol therefore routes to all the active nodes are discovered in advance. The information about the entire routing path is transmitted to all the active nodes after a fixed interval of time- regardless of any route changes [16]. Proactive protocol does not only generate high overheads but also consumes bandwidth because of its proactive nature, so it is not fit for network with high mobility and high node density. A common type of proactive protocol is DSDV.

\section{Simulation Model}

Detailed simulation model based on NS-2 is used in our evaluation. NS2 is an event driven, object oriented network simulator that allows the simulation of many ad-hoc networks. It implements traffic sources, network protocols and queue management mechanisms. NS2 is written in two different languages (C++ and OTCL) in order to separate control and data path implementations.

$\mathrm{C}++$ is used for detailed protocol implementation because of its speed while OTCL, though runs slowly, is used in configuration and operation because of its flexibility - it can be modified very fast thus suitable.

In our simulation, two stationary source nodes (scrNode) were discretely run on UDP and TCP to send "rescue messages" to other two fixed destinations (dstNode). The scenarios were very chaotic with intermediate mobile nodes moving randomly at high speeds from random positions to random destinations.

Pause time is zero which means that the intermediate nodes move erratically throughout the simulation time. The simulation time is deliberately short to enable us determine the suitable transport and routing protocol grouping that will deliver an emergency message timely, intelligently and reliably.

We arranged the grouping of the protocols as shown below:

Table 1: Routing protocol/Transport protocol

\begin{tabular}{|l|l|l|l|}
\hline AODV/UDP & AOMDV/UDP & DSR/UDP & DSDV/UDP \\
\hline AODV/TCP & AOMDV/TCP & DSR/TCP & DSDV/TCP \\
\hline
\end{tabular}




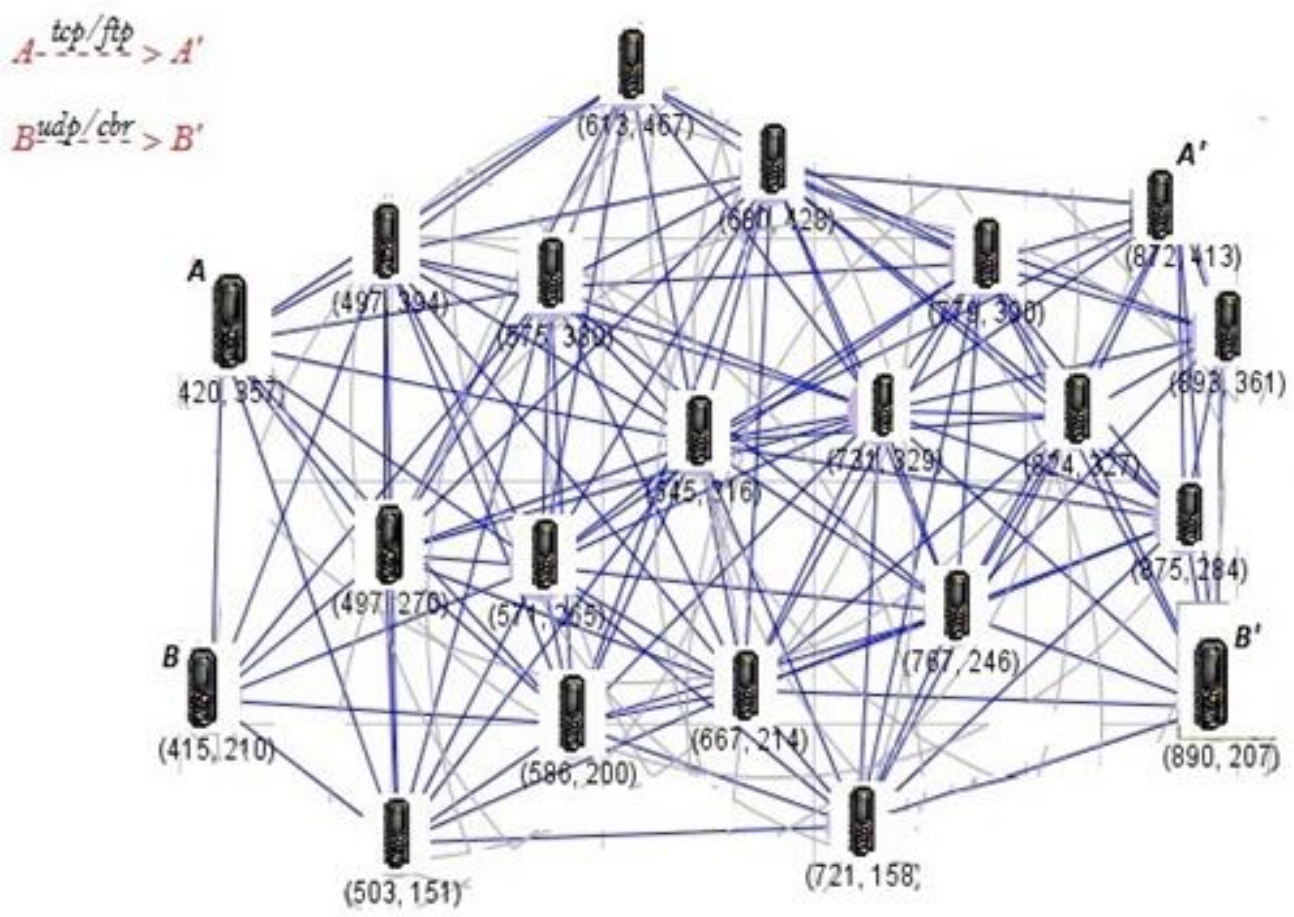

Figure 1: Pictorial Representation of our 20 nodes network model

The network for simulations consists of $10,20,30,40,50,60,70,80,90,100$ mobile nodes with four nodes of each simulation run fixed. Two of the fixed nodes were the source nodes implemented on TCP and UDP protocols and the other stationary nodes are the destination nodes as shown in figure 1 .

Traffic was generated using constant bit-rate (CBR) - which is attached to UDP; and file transfer protocol (FTP) - attached to TCP. Packet size is 1500 bytes. Table 1 shows the simulation parameters.

Table 2: Simulation Parameters for node configuration

\begin{tabular}{ll}
\hline Parameters & \multicolumn{1}{c}{ Value } \\
\hline Channel Size & Channel/WirelessChannel \\
Number of Nodes & $10,20,30,40,50,60,70,80,90,100$ \\
Source Traffics & CBR, FTP \\
Antenna Model & Antenna/OmniAntenna \\
Radio Propagation Model & Propagation/TwoRayGround \\
Node Speed & $0 \mathrm{~m} / \mathrm{s}-70 \mathrm{~m} / \mathrm{s}$ (random) \\
Number of simulated scenarios & 40 \\
Interface Queue Type & Queue/Droptail/PriQueue \\
Packet Size & 1500 \\
MAC Type & Mac/802.11 \\
Buffer Size & 50 \\
Transport Layers & UDP, TCP \\
Simulation time & $20 \mathrm{~s}$ \\
\hline
\end{tabular}




\section{Simulation Results and Performance Assessment}

Figure 2 shows the routing path for the TCP transport connection for AODV and AOMDV. Since the network nodes are constantly moving, when an intermediate node using AODV protocol goes out of range or exceeds is lifetime, the upstream node quickly sends link failure message to other active upstream nodes which re-propagate the message till it gets to the source node which then initiates fresh route discovery. Unlike AOMDV which has multiple source-destination routes, the single source-destination route in AODV makes it search continuously for fresh route when there is link breakage.
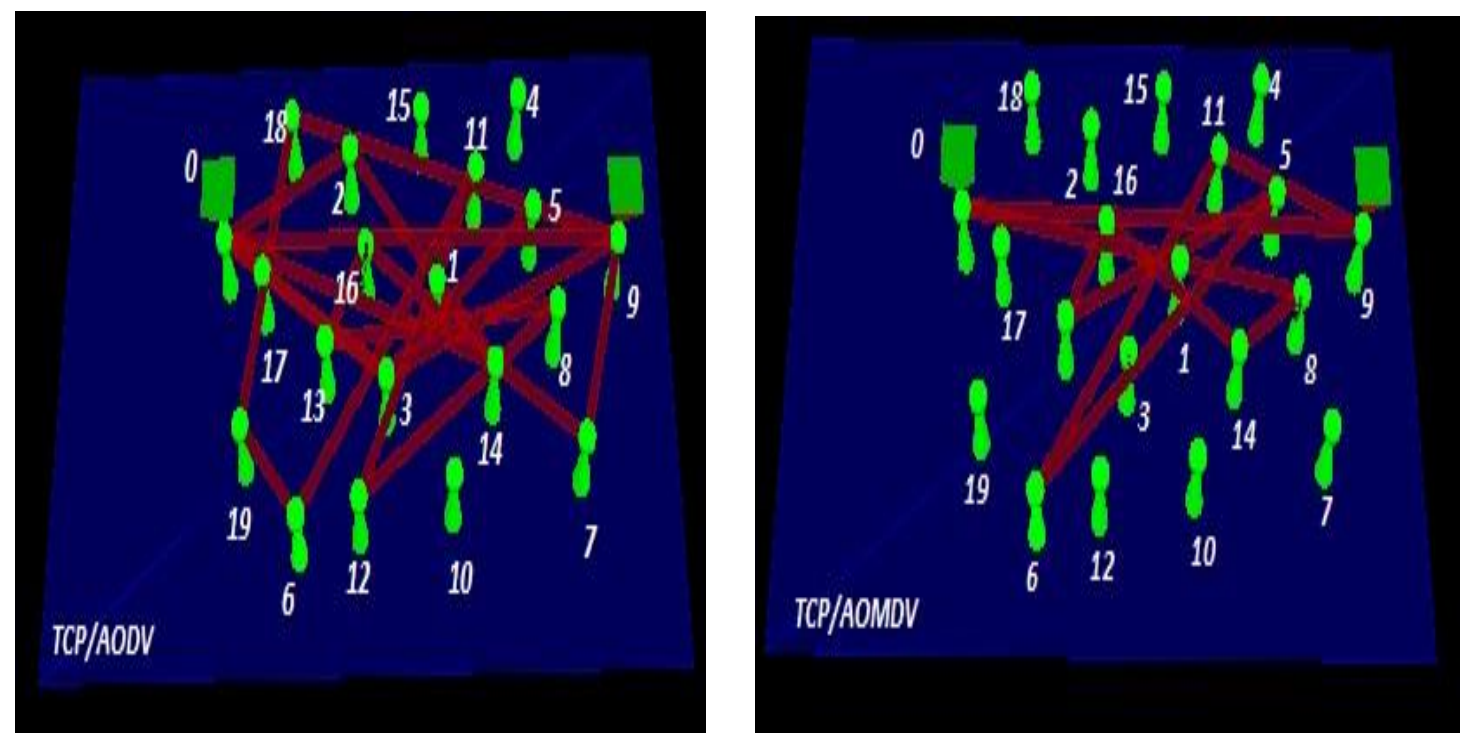

Figure 2: AODV \& AOMDV Routing Path for TCP connection

AODV route path $\rightarrow$ route 1: 0-2-1-14-7-9; route 2: 0-9; route 3: 0-17-3-9; route 4: 0-17-3-5-9; route 5: 0-14-16-13-8-12-11-6-19-18-9. AOMDV route path $\rightarrow$ route $1: 0-9$; route $2: 0-8-14-16-$ $13-5-6-11-9$

Forty simulations were run for both simple and complex multi-hop network. For each scenario, UDP and TCP protocols were set up under the same network conditions of mobility, packet load and density. AODV, AOMDV, DSR and DSDV were individually implemented on two transport connections. Average delay, PDR, delay jitter and throughput were calculated for the routing and transport protocol grouping.

(i) Average end to end delay: It is defined as the total time taken by the source node to deliver packets to the destination node. This includes possible delays caused during route discovery, delay at the interface queue, retransmission delays and propagation delay.

Mathematically, delay time resulting from discovery intermediate nodes is:

$$
D_{N D}=\sum_{i=1}^{n}\left(T_{P R H M}-T_{B H M}\right)
$$

Where $\mathrm{D}_{\mathrm{ND}}=$ delay in route discovery, $\mathrm{T}_{\mathrm{PRHM}}=$ processing and returning period of Hello message and $\mathrm{T}_{\mathrm{BHM}}=$ broadcast start time of hello message. 
While delay caused from processing control packets including RREQ, RREP and RERR is represented by:

$$
D_{P D}=\left(T_{R R E P}-T_{R R E Q}\right)
$$

Where $\mathrm{D}_{\mathrm{PD}}=$ delay due to packet processing, $\mathrm{T}_{\mathrm{RREP}}=$ receiving time of propagation and $\mathrm{T}_{\mathrm{RREQ}}=\mathrm{RREQ}$ transmission time. Therefore, end-to-end delay $D_{E E}$ can be represented by:

$$
D_{E E}=D_{N D}+D_{P D}
$$

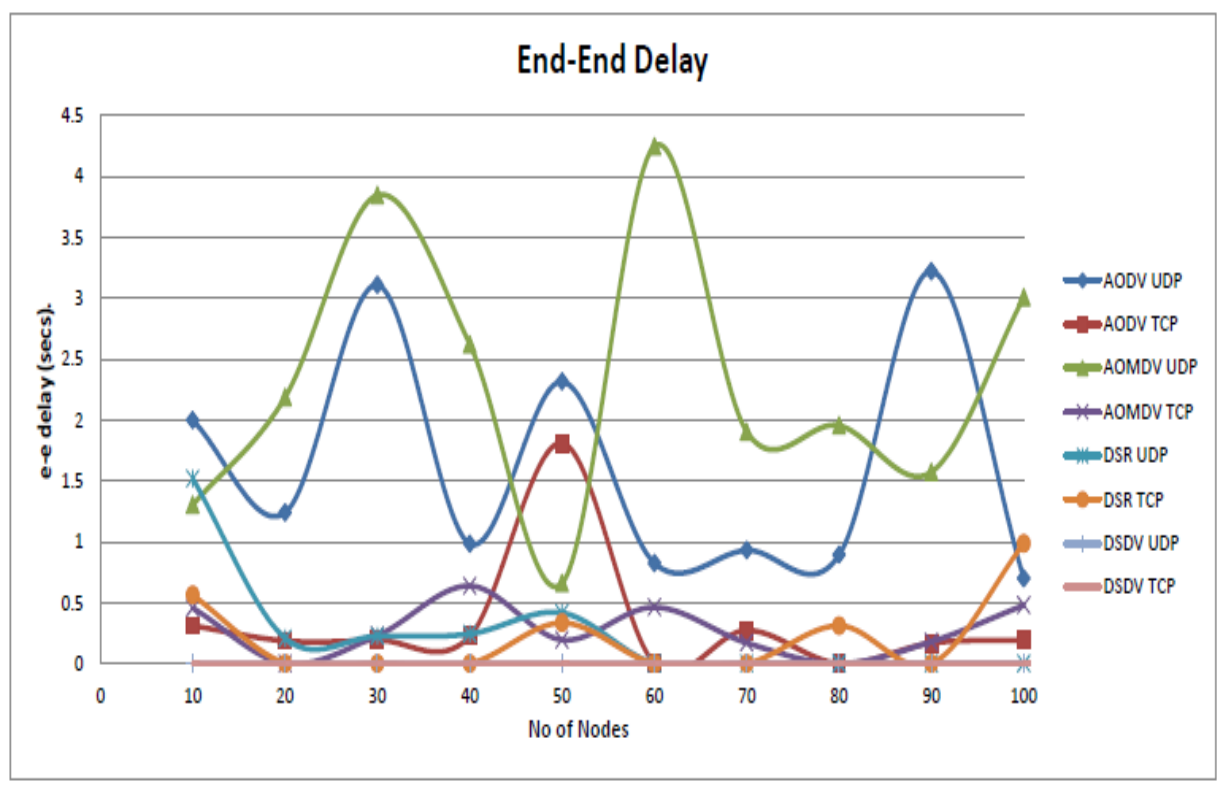

Figure 3: Average end-end delay for all protocol groupings

From figure 3, AOMDV/UDP performed most badly compare to the other protocols in end-to-end delay. This is because in the event of link breakage, AOMDV attempts to establish an alternate path from the backup routes which thus generates extra delay and also UDP which works with CBR continuously produces packets "un-adaptively" this also causes delay due to queuing at the interface therefore UDP protocol has high delay for all the reactive protocols.

AODV/TCP maintained almost a constant delay before having a sharp delay increase when the number of nodes increased to 50 but later normalized.

This is because AODV repairs link failure by choosing a fresh route and speedily re-establishes connection. In order to deliver the packets to the destination node, AODV/TCP used five different routes (figure 2). DSR/TCP performs better but the delay increases with node density. DSDV performed very well in end-end delay however, figure 4 shows that DSDV is not suitable for network with short connection time, large packet size, high mobility and high node density because of its proactive nature.

For most of the node numbers, DSDV grouping could not establish connection. Figure 4 shows the route messages generated in node number 50 by some of the active nodes (represented as srcNode in figure 4) advertising their position in the network. However, the advertisement was not successfully delivered to the destinations (dstNode). DSDV does not have route discovery mechanism, therefore, packets will be dropped if route does not exist or simulation time is over. Thus, DstNode $=-1$ that is timeout. 

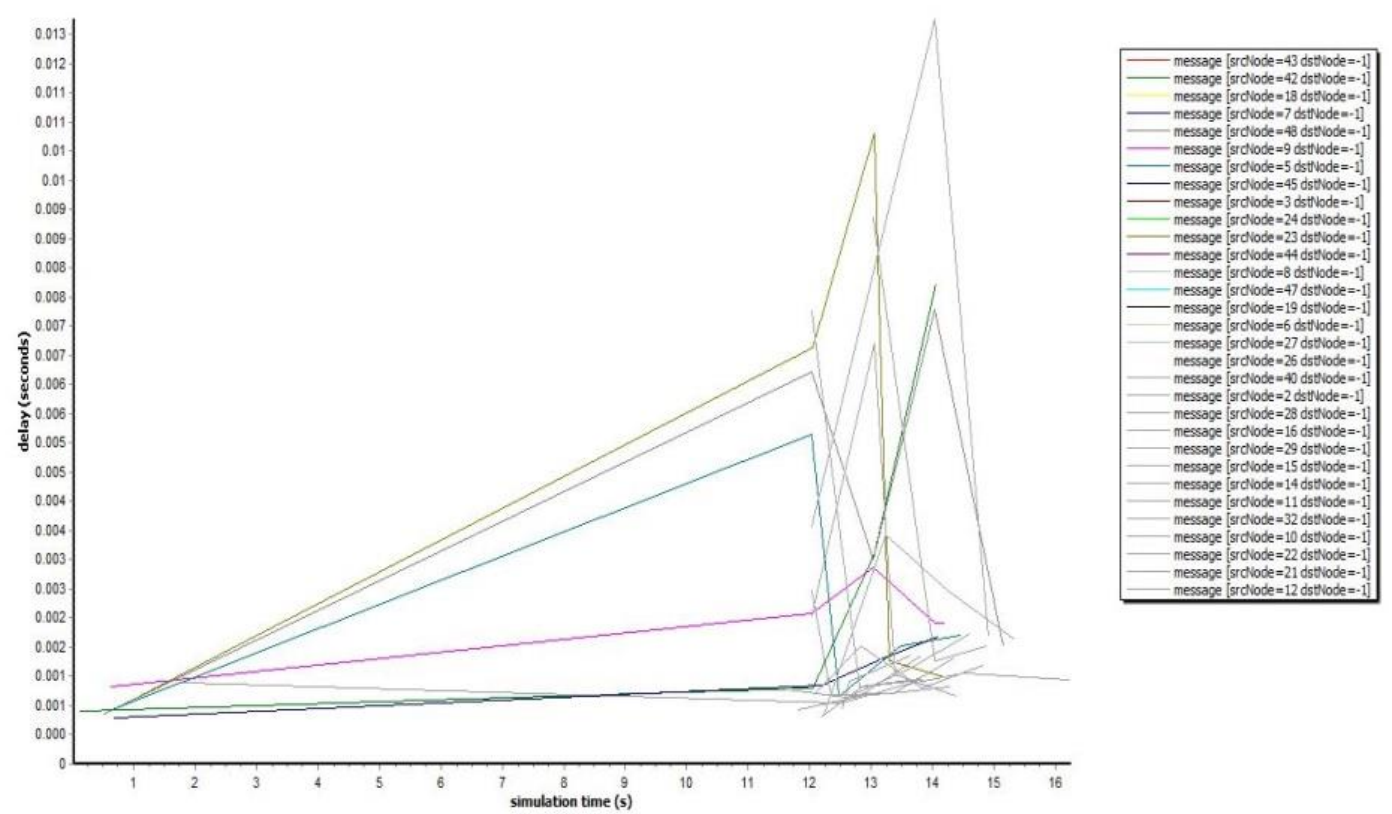

Figure 4: Delay in broadcasting route-table messages in DSDV

(ii) Throughput: This is the number of packets the destination node $\mathrm{D}$ gets from source $\mathrm{S}$ divided by the total time used by $\mathrm{D}$ to accept the last packet. Throughput is a function of other factors such as collision, congestion, loss etc. Its unit is bits per second.

Mathematically,

$$
T=\frac{N_{\text {out }} \cdot L_{F} \cdot 8}{T_{t}}
$$

Where $\mathrm{T}$ is throughput, $\mathrm{N}_{\text {out }}=$ amount of frame to send, $\mathrm{L}_{\mathrm{F}}=$ length of frame and $\mathrm{Tt}=$ testing period and $8=$ conversion from bytes to bits

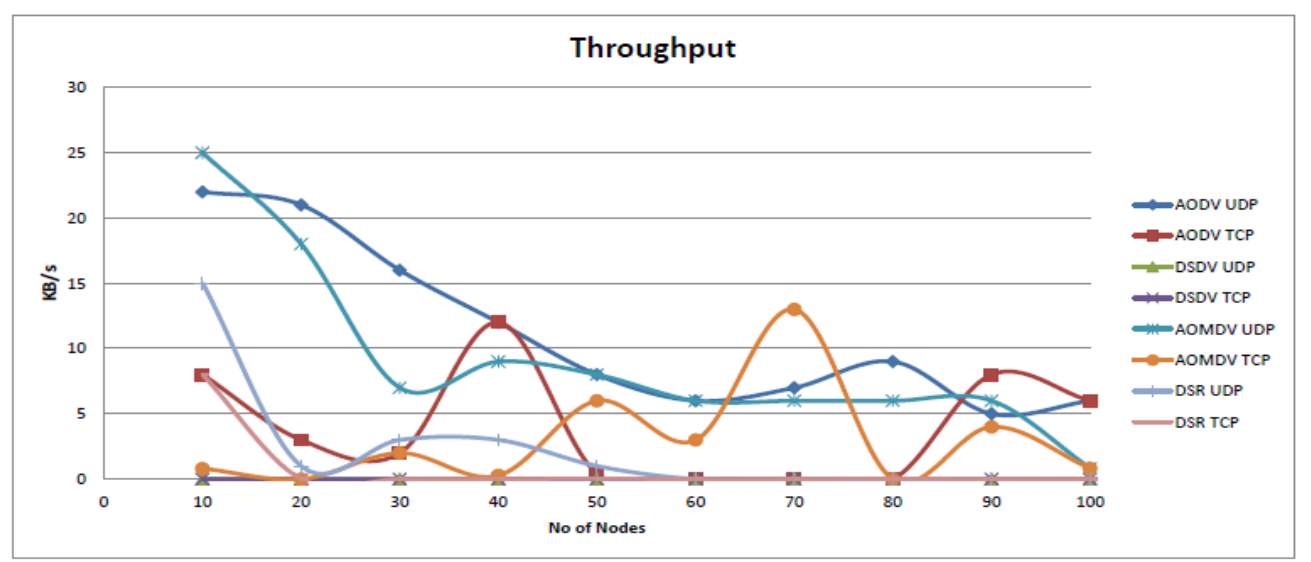

Figure 5: Throughput for all the protocol groupings

As presented in figure 5, TCP performed worse than UDP in throughput since the default configurations of TCP has been consciously designed to give up throughput for fair bandwidth sharing on congested networks [17]. From our simulation result, AODV/UDP and AOMDV/UDP 
performed better than other grouping and AOMDV/UDP maintained a constant throughput for node 60 to 90 .

More so, we observed that for all the groupings, number of node increase leads to throughput decrease as increase in nodes creates huge congestion hence the increase in propagation time for the packet.

(iii) Packet Delivery Ratio (PDR): the ratio of packets delivered at the destination node to those generated in the application layer of the source node.

Mathematically, PDR is:

$$
P D R=\sum_{i=1}^{n} \frac{P_{D}}{P_{S}} \times 100
$$

Where $\mathrm{P}_{\mathrm{D}}=$ packets received by destination node,

$\mathrm{P}_{\mathrm{S}}=$ packets generated by source $\mathrm{S}$ and $\mathrm{n}=$ no of nodes

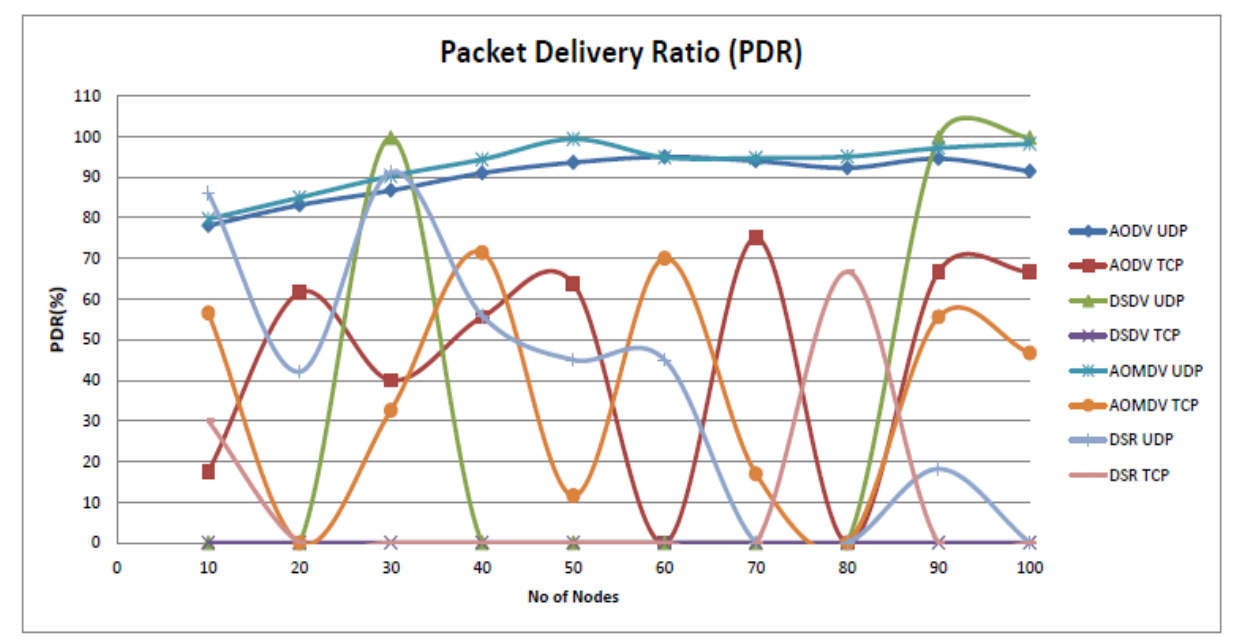

Figure 6: PDR for all the protocol groupings

For simple network, PDR performed well in DSR/UDP compare to other protocols but when the nodes increase, the performance decreases. For AODV and AOMDV on UDP, packet delivery ratio increases when network density increases. In general, UDP/CBR transport protocol performs better than TCP/FTP for all the routing protocols for PDR metric. It's because connection-oriented TCP has a retransmission mechanism that enables it wait for earlier packets to be delivered before transmitting new packets unlike UDP that is connectionless and transmits packets in a "fire and forget" manner therefore having higher delivery ratio. AOMDV performed better than other routing protocols because its intermediate nodes have alternate paths thus they can easily reconfigure themselves when there is connection breakage- thereby mitigating route discovery process and also increasing packet delivery.

DSDV/UDP performed well at nodes 30, 90 and 100.

(iv) Delay Jitter: Network state is always fluctuating and dataflow sometimes is huge and sometimes small. When the flow is large some packets will have to queue in the node to be delivered at the time network state is small, therefore the sending period for the transmitted packets from source node to the destination node will not be the same. The difference is called Delay Jitter. The more the jitter the more unstable the network, and the more the loss of QoS. 


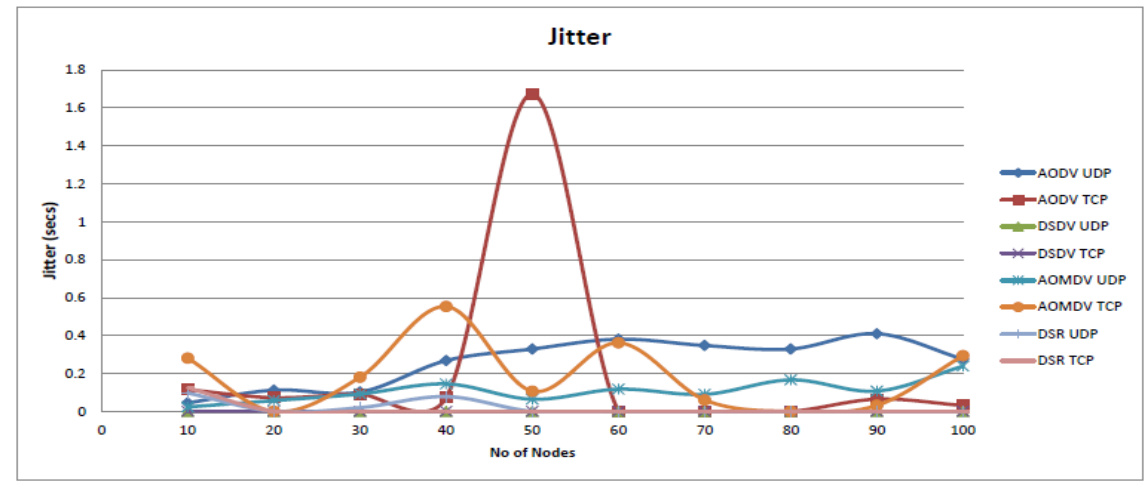

Figure 7: Delay Jitter for all the simulated scenarios

From our simulation result shown in Figure 7, the protocols maintain a low level of delay jitter between 0 to 0.6 secs except AODV/TCP which has a sharp increase at node 50; the effect of this is also noticeable in end to end delay at node 50. AOMDV/UDP maintains almost a constant delay jitter when number of nodes increase. AOMDV/TCP shows the maximum deviation. It can be observed that at node number 80 , TCP connection did not generate any delay jitter. This is because there was no data connection in AOMDV/TCP (dstNode $=-1$ (timeout)) due to failure in RREQ as depicted in figure 7 and failure of RREP connection in AODV/TCP and DSR/TCP as shown in figure 8 and 9.

Note that figure $8,9 \& 10$ show the delay jitter generated during route discovery process and other form of connection establishing activities for node number 80 .

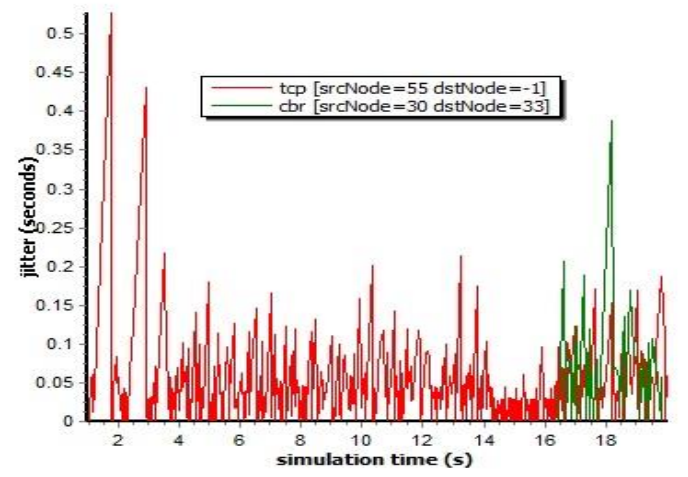

Figure 8: Delay jitter for 80 nodes network in AOMDV

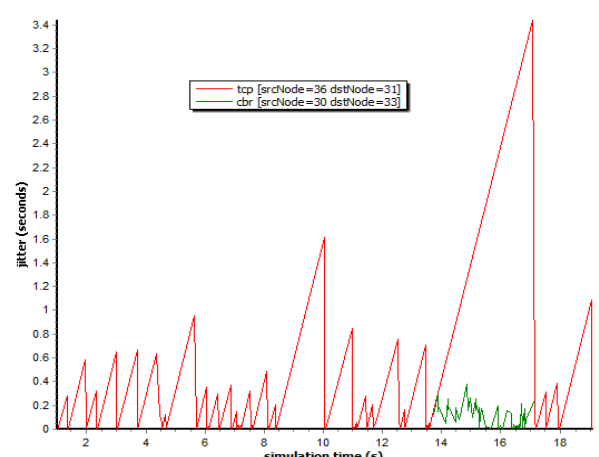

Figure 9: Delay jitter for 80 nodes network in

AODV

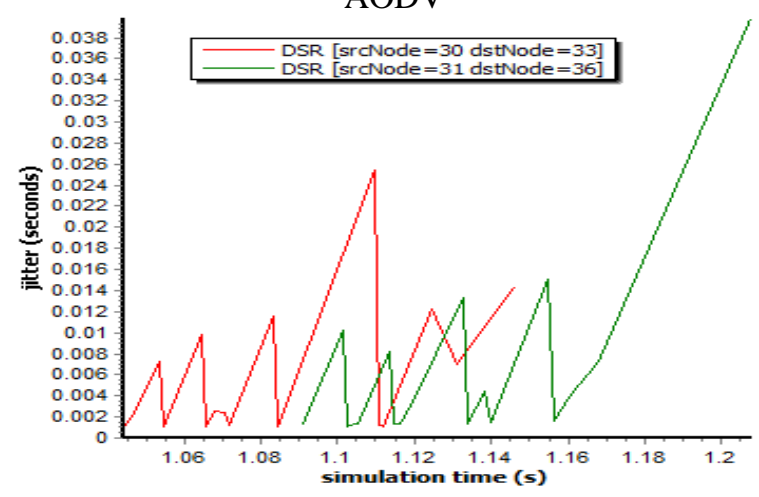

Figure 10: Delay Jitter for 80 nodes network DSR 


\section{CONCLusion}

UDP and TCP transport protocols' performances were carefully studied on four ad-hoc routing protocols: AODV, DSR, DSDV and AOMDV in other to determine the protocol grouping that will deliver a "distress message" in a timely, intelligent and reliable manner in a simple and complex scenario. Several simulations were run using NSG2.1 software to generate TCL file that is then run on NS2.34 to generate tr. file. The two files were analyzed using NAM, Microsoft Excel and NS2 Visual Tracer Analyzer 0.2.72 software. Throughputs, end to end delay, PDR and delay jitter were assessed under both low and high traffic condition.

Simulation results show that UDP performed poorly in end to end delay and delay jitter with the routing protocols but outperformed TCP in both throughput and PDR. This is because UDP has no flow control mechanism and packets are delivered in a "fire and forget" manner.

Delay in TCP is low however congestion controls for example: congestion window and slow-start threshold give TCP low throughput and PDR.

Reactive protocols achieved better performance than the proactive protocol. We observed that connection was not established by DSDV/TCP grouping- the route table messages generated did not get to the destination nodes as presented in figure 4 due to low simulation time, high node mobility and network density. AOMDV and AODV performed well in PDR- the more the nodes the higher the PDR while in throughput, we observed that the higher the number of nodes the lower the throughput for AOMDV/UDP, AODV/UDP and DSR/UDP.

\section{REFERENCES}

[1] K. Solomon, T.J Edem and S. Gawusu. 2017. Comparative Analysis and Examination of Hybrid Multi-Meshed Tree (HMMT) Routing Protocol for MANET. ICCMA 2017, Edmonton.Canada. DOI: http://doi.org/10.1145/3149827.3149828 Pp 114-119.

[2] T.K Saini and M.K. Dhaka. 2009, Performance Simulation of Tahoe, Reno, New Reno and SACK over Terrestrial and Geostationary Satellite Links. IEEE International Conference on Methods and Models in Computer Science, Delhi, India. DOI: 10.1109/ICM2CS.2009.5397980.

[3] J. Zhu and T. Bai. Performance of Tahoe, Reno and SACK TCP at Different Scenarios, IEEE International Conference on Communication technology, Guilin, China. DOI: 10.1109/ICCT.2006.341884.

[4] S. Gharge and A. Valanjoo. 2014. Simulation Based Performance Evaluation of TCP Variants and Routing Protocols in Mobile Ad-Hoc Networks. IEEE International Conference on Advances in Engineering \& Technology Research (ICAETR-2014). India. DOI: 10.1109/ICAETR.2014.7012810.2014.

[5] A. Boudhir and W Archraf. 2017. Study of the Impact of Load and Density on the Behavior of Routing Protocols AODV and DSDV in the VANET Networks. SCAMS-ACM. Morroco. DOI: $10.1145 / 3175628.3175629$.

[6] B.M Susanto, A. Hariyanto and Surateno. 2017. Performance Comparison of MANET Routing Protocol based on Random Waypoint Mobility Model. CSAI-ACM. Indonesia. DOI: http://doi.org/10.1145/3168390.3168403.

[7] N. Rathod and N Dongre. 2017. MANET Routing Protocol Performance for Video Streaming. 2017 International Conference on Nascent Technologies in the Engineering Field (ICNTE-2017). Pp 1-5 
[8] H.P Wang and L. Cui. An Enhanced AODV for Mobile Ad Hoc Network. Proc. Seventh International Conference on Machine Learning and Cybernetics. Kunming. China. Pp. 1-2.

[9] C. Perkins, E. Belding-Royer and S. Das. 2003. Ad-Hoc On-Demand Vector (AODV) Routing. Network Working Group, RFC3561, IETF MANET Working Group.

[10] Harris Simaremare et al, 2013. Performance Comparison of Modified Reference Point Group Mobility and Random Waypoint Mobility Models. IEEE ICC 2013- Next Generation Networking Symposium.

[11] K. Mahajan, M.A. Rizvi, D.S Karaulia. 2014. Path Optimization of AODV by EO-AODV in MANET. ACM-ICTCS '14 Proceedings of the International Conference on Information and Communication Technology for Competitive Strategies.

[12] Vinay R., Sandeep K.A., Mano Y, 2016. Performance of AODV protocol for H-MANETs. IEEE 2016 International Conference on Advances in Computing, Communication \& Automation (ICACCA).

[13] R.B Patel. Ad Hoc On-Demand distance Vector Routing. http://www.srmuniv.ac.in/ramapuram/sites/ramapuram/files/MANET-AODV.pdf.

[14] V. Bondre and S. Dorle. 2015. Design and Performance Evaluation of AOMDV Routing Protocol for VANET. IEEE International Conference on Computer, Communication and Control (IC4-2015). DOI: 10.1109/IC4.2015.7375523.

[15] D.B Johnson, D.A. Maltz and J Broch. DSR: The Dynamic Source Routing for Multi-Hop Wireless Ad-Hoc Networks. Computer Science Department, Carnegie Mellon University. Pittsburg PA 15213 3891. Pp 4-6.

[16] N. Patel, A. Pawar and N. Shekokar, 2015. A Survey on Routing Protocols for MANET. International Journal of Computer Applications (0975-8887). Volume 110- No. 11.

[17] S. Giannoulis, C Antonopoulos, E. Topalis, A. Athanasopoulos, A Prayati and S. Koubias. 2009. TCP vs. UDP Performance Evaluation for CBR Traffic On Wireless Multihop Networks.

[18] Vinay R., Sandeep K.A., Mano Y, 2016. Performance of AODV protocol for H-MANETs. IEEE 2016 International Conference on Advances in Computing, Communication \& Automation (ICACCA)

\section{AUTHORS}

Adebayo Seyi is a final year post graduate student of the department of information and communication engineering, China University of Mining and Technology, China. His research areas include mobile ad-hoc networks, network protocols and D2D communication.

Taiwo Blessing Ogunseyi currently pursuing his $\mathrm{Ph} . \mathrm{D}$. degree in Information Security with the College of Information Engineering, Communication University of China, Beijing China. His research interests include wireless network security, applied cryptography, user privacy and privacy preserving data mining. He is a student member of IEEE.
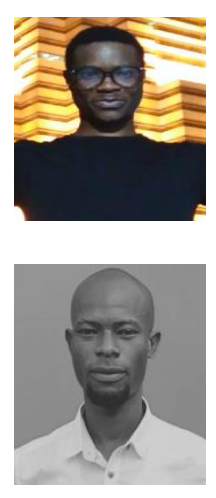